\title{
Functional magnetic resonance imaging and control over the biceps muscle after intercostal-musculocutaneous nerve transfer
}

\author{
Martijn J. A. Malessy, M.D., Ph.D., Dick Bakker, M.D., Ad J. DekKer, Ph.D., \\ J. Gert van Dijk, M.D., Ph.D., And Ralph T. W. M. Thomeer, M.D., Ph.D. \\ Departments of Neurosurgery, Radiology and Neurology, and Clinical Neurophysiology, Leiden \\ University Medical Center, Leiden, The Netherlands
}

\begin{abstract}
Object. Recent progress in the understanding of cerebral plastic changes that occur after an intercostal nerve (ICN)musculocutaneous nerve $(\mathrm{MCN})$ transfer motivated a study with functional magnetic resonance (fMR) imaging to map reorganization in the primary motor cortex.

Methods. Eleven patients with traumatic root avulsions of the brachial plexus were studied. Nine patients underwent ICN-MCN transfer to restore biceps function and two patients were studied prior to surgery. The biceps muscle recovered well in seven patients who had undergone surgery and remained paralytic in the other two patients. Maps of neural activity within the motor cortex were generated for both arms in each patient by using fMR imaging, and the active pixels were counted. The motor task consisted of biceps muscle contraction. Patients with a paralytic biceps were asked to contract this muscle virtually. The location and intensity of motor activation of the seven surgically treated arms that required good biceps muscle function were compared with those of the four arms with a paralytic biceps and with activity obtained in the contralateral hemisphere regulating the control arms.

Activity could be induced in the seven surgically treated patients whose biceps muscles had regained function and was localized within the primary motor area. In contrast, activity could not be induced in the four patients whose biceps muscles were paralytic. Neither the number of active pixels nor the mean value of their activations differed between the seven arms with good biceps function and control arms. The weighted center of gravity of the distribution of activity also did not appear to differ.

Conclusions. Reactivation of the neural input activity for volitional biceps control after ICN-MCN transfer, as reflected on fMR images, is induced by successful biceps muscle reinnervation. In addition, the restored input activity does not differ from the normal activity regulating biceps contraction and, therefore, has MCN acceptor qualities. After ICN-MCN transfer, cerebral activity cannot reach the biceps muscle following the normal nervous system pathway. The presence of a common input response between corticospinal neurons of the ICN donor and the MCN acceptor seems crucial to obtain a functional result after transfer. It may even be the case that a common input response between donor and acceptor needs to be present in all types of nerve transfer to become functionally effective.
\end{abstract}

\section{KEY WORDS • brachial plexus • functional magnetic resonance imaging • intercostal nerve $\bullet$ motor cortex $\bullet$ nerve transfer $\bullet$ plasticity}

$\mathrm{I}$ $\mathrm{N}$ severe brachial plexus traction lesions including C-6 root avulsions, the transfer of ICNs to the MCN can be applied to regain biceps function. ${ }^{19}$ In this technique, the third through fifth ICNs are dissected free, transected close to the sternum, tunneled to the axilla, and coapted to the MCN.

Normally, corticospinal neurons that ultimately project to the intercostal muscles are active during the execution of motor programs for respiration and/or posture control, effected through intercostal motor neurons and muscles.

After transfer, central ICN motor programs for respiration and posture control become connected to the biceps muscle. Initially, biceps contraction can only be effected by means of a voluntary respiratory effort. At the end stage of

Abbreviations used in this paper: $\mathrm{fMR}=$ functional magnetic resonance; $\mathrm{ICN}=$ intercostal nerve; $\mathrm{MCN}=$ musculocutaneous nerve; $\mathrm{SD}=$ standard deviation; TMS = transcranial magnetic stimulation. reinnervation, however, volitional control over biceps muscle contraction can be achieved without resorting to respiratory effort. ${ }^{22}$ This change in motor control requires a central adaptation to altered peripheral connections..$^{20,24}$

We previously used TMS to study the cerebral plastic changes that occur after ICN-MCN transfer. Our studies involving TMS mapping in healthy volunteers revealed that the cortical map of the intercostal cortical area is localized in the midline, whereas that of the biceps muscle is located a few centimeters lateral from the midsagittal plane. The map coordinate system used in that study was the international 10-20 electrode system for electroencephalography examinations, which refers to skull landmarks. ${ }^{12,21,33}$ At the end stage of reinnervation, the center of gravity of TMS maps of the reinnervated biceps muscle differed from that of the medial location of the intercostal muscles. Surprisingly, it was indistinguishable from that of the normal location of the lateral biceps muscle. We hypothesized that the 
TABLE 1

Clinical characteristics of patients*

\begin{tabular}{|c|c|c|c|c|c|c|}
\hline \multirow[b]{2}{*}{ Case No. } & \multirow[b]{2}{*}{$\begin{array}{l}\text { Age }(y r s) \\
\text { Sex }\end{array}$} & \multirow[b]{2}{*}{ Side/Diagnosis } & \multirow[b]{2}{*}{ Reconstruction } & \multicolumn{2}{|c|}{ Interval (mos) } & \multirow[b]{2}{*}{$\begin{array}{l}\text { Biceps } \\
\text { Force } †\end{array}$} \\
\hline & & & & $\begin{array}{l}\text { Trauma } \\
\text { to Surgery }\end{array}$ & $\begin{array}{l}\text { Surgery to } \\
\text { fMR Imaging }\end{array}$ & \\
\hline 1 & $16, \mathrm{M}$ & rt/av C5-T1 & $\begin{array}{l}\text { ICN 3,4,5-MCN transfer, } \\
\text { ICBN-LDMN transfer }\end{array}$ & 2 & 103 & 4 \\
\hline 2 & $16, \mathrm{M}$ & rt/av C5-T1 & ICN 4,5-MCN transfer & 2 & 98 & 4 \\
\hline 3 & $33, \mathrm{~F}$ & $\begin{array}{l}\mathrm{rt} / \mathrm{IFNT} \text { C-5, } \\
\text { av C6-7 }\end{array}$ & $\begin{array}{l}\text { ICN 4,5,6-MCN transfer, } \\
\text { AcN-SSN transfer }\end{array}$ & 5 & 84 & 4 \\
\hline 4 & $16, \mathrm{M}$ & $1 \mathrm{t} / \mathrm{av} \mathrm{C} 5-\mathrm{T} 1$ & $\begin{array}{l}\text { ICN 3,4,5,6-MCN transfer, } \\
\text { ICN 2-MPN transfer } \\
\text { AcN-SSN transfer }\end{array}$ & 2 & 68 & 4 \\
\hline 5 & $22, \mathrm{~F}$ & $\begin{array}{l}\text { rt/av C5-7, } \\
\text { partial NT of } \\
\text { CN XI }\end{array}$ & ICN 4,5,6-MCN transfer & 2 & 63 & 4 \\
\hline 6 & $18, \mathrm{M}$ & $\begin{array}{l}\text { 1t/IFNT C5, } \\
\text { av C6-T1 }\end{array}$ & $\begin{array}{l}\text { ICN 3,4,5-MCN transfer, } \\
\text { C4-LDMN transfer }\end{array}$ & 1 & 46 & 4 \\
\hline 7 & $19, \mathrm{~F}$ & rt/av C5-7 & $\begin{array}{l}\text { ICN 3,4,5-MCN transfer, } \\
\text { HN-AN transfer, AcN-SSN transfer }\end{array}$ & 1 & 45 & 4 \\
\hline 8 & $22, \mathrm{M}$ & $\begin{array}{l}\text { 1t/partial av C-5, } \\
\text { av C6-T1 }\end{array}$ & $\begin{array}{l}\text { ICN 3,4,5-MCN transfer, } \\
\text { graft of C5-SCN to LDMN }\end{array}$ & 1 & 73 & 0 \\
\hline 9 & $20, \mathrm{M}$ & $\begin{array}{l}\text { 1t/NT C-5, } \\
\text { av C6-T1 }\end{array}$ & $\begin{array}{l}\text { ICN 3,4,5 \& MCN transfer, } \\
\text { graft of C-5 \& SCN to LDMN }\end{array}$ & 3 & 69 & 0 \\
\hline 10 & $16, \mathrm{M}$ & $\begin{array}{l}\text { 1t/IFNT C-5, } \\
\text { av C6-T1 }\end{array}$ & ICN 3,4,5-MCN transfer & 3 & prior & 0 \\
\hline 11 & $44, \mathrm{~F}$ & $\begin{array}{l}\mathrm{rt} / \mathrm{NT} \mathrm{C}-5, \\
\quad \text { av C6-T1 }\end{array}$ & graft of C5-VDST & 4 & prior & 0 \\
\hline
\end{tabular}

* $\mathrm{AN}=$ axillary nerve; av = avulsion; $\mathrm{AcN}=$ accessory nerve; $\mathrm{HN}=$ hypoglossal nerve; $\mathrm{ICBN}=$ intercostobrachial nerve; IFNT = intraforaminal neurotmesis; LDMN = lateral division of median nerve; MPN = medial pectoral nerve; SCN = supraclavicular nerve; $\mathrm{SSN}=$ suprascapular nerve; VDST = ventral division of superior trunk.

$\dagger$ Medical Research Council grade.

ICN-MCN reinnervated biceps map represents a cortical network of flexion interneurons that connect to the medially located intercostal corticospinal neurons..$^{21}$ There was a difference, however, between TMS maps of ICN-MCN reinnervated biceps and that of control muscles: the cortical area of the ICN-reinnervated biceps was less excitable and smaller than that of the healthy biceps..$^{21}$ Transcranial magnetic stimulation excites pyramidal tract cells, either directly or indirectly. ${ }^{11}$ The TMS mapping, therefore, reveals the anatomical connection involved in neural output activity. The observed plastic phenomena in the neural output activity after ICN-MCN transfer appear to be located approximately $2.8 \mathrm{~cm}$ in front of and $3.5 \mathrm{~cm}$ lateral to the central vertex position in the midsagittal line..$^{21}$ It is highly likely that this involves the primary motor cortex, because the position of the central sulcus is located close to the central vertex position. ${ }^{12}$

Until now, it is unknown whether plastic changes are limited to neural output activity. Theoretically, plastic changes in the neural input activity to the cortex may also take place to enable the change of control over the ICN-MCN reinnervated biceps muscle.

Functional magnetic resonance imaging has been used to map neuronal activity within the brain during contraction of the biceps muscle. ${ }^{27}$ Recently, the relationship between the fMR imaging signal and the underlying neural activity has become more clear. ${ }^{17} \mathrm{~A}$ spatially restricted increase in the fMR imaging signal directly reflects an increase in neural activity. An analysis of the activation of an area of the brain, as seen on fMR images, showed that the signal actually re- flects the neural activity related to the input and the local intracortical processing, rather than the output of the area.

In the present study we used fMR imaging to study possible plastic changes in the input neural activity of voluntary biceps contraction after ICN-MCN transfer by analyzing the signal intensity in the primary motor cortex.

\section{Clinical Material and Methods}

\section{Patient Population}

Eleven patients with root avulsions following a traction lesion of the brachial plexus were included in the fMR imaging study. All participants gave informed consent. Nine patients underwent ICN-MCN transfer and the biceps muscle recovered well in seven. Two patients were studied before surgery (their surgeries were performed recently and no follow-up data are available). The mean age of the patients $( \pm \mathrm{SD})$ at the time of injury was $22 \pm 8.8$ years (median 19 years) and the mean interval ( \pm SD) between trauma and surgery was $2.4 \pm 1.3$ months (median 2 months). Characteristics of the patients are summarized in Table 1 . Four of the five patients who were examined with TMS cooperated in the fMR imaging study as well. ${ }^{21}$

Before surgery, needle electromyography studies had been performed to document a complete absence of activity during maximum voluntary effort, and computerized tomography myelography was used to detect root avulsions. In all cases, the entire trajectory of the brachial plexus had been exposed during surgery ${ }^{32}$ and root avulsion was es- 
tablished with certainty by the absence of a spinal nerve within the intervertebral foramen. In four patients there was avulsion of the C5-T1 nerve roots and in two patients there was avulsion of the C5-C7 roots. In another five patients the $\mathrm{C}-5$ nerve had ruptured and there was avulsion of the C-6 and C-7 nerve roots; in four of these patients there was additional avulsion of the $\mathrm{C}-8$ and $\mathrm{T}-1$ roots. Further resection of four of the ruptured C-5 stumps up to the intervertebral foramen demonstrated only a fibrotic appearance to the stumps with less than $50 \%$ myelin, which was confirmed by examination of a frozen section obtained intraoperatively. ${ }^{23}$ Intraforaminal neurotmesis of the C-5 stump made these patients ineligible for nerve grafting to restore biceps muscle function, which otherwise would have been preferable. We previously described the surgical technique of the ICNMCN transfer. ${ }^{19}$ Briefly, the third through fifth ICNs were dissected free, transected as closely as possible to the sternum, and tunneled to the axilla. The MCN was cut and these ICNs were coapted directly to the MCN. Postoperative electromyography studies revealed typical respiratory activity in the biceps muscle ${ }^{22}$ of the seven patients with good recovery, which left no doubt that the reinnervation was due to the surgical repair. Action potentials remained absent in the biceps muscle of the two surgically treated patients with a paralytic biceps. Before each fMR imaging study, the force exerted by the reinnervated biceps muscle was assessed according to the Medical Research Council scale. $^{31}$

The preoperative fMR imaging studies obtained in two patients were performed 1 or 2 weeks before surgery. The fMR imaging studies obtained in patients following surgery were carried out at least 45 months after surgery, ensuring that functional restoration was complete at this time. All patients with successful biceps reinnervation exhibited conscious volitional control over biceps contraction at the time of fMR imaging, including the ability to contract the biceps muscle during continuous breathing. ${ }^{22}$

\section{Magnetic Resonance Imaging Studies}

A 1.5-tesla Advanced Clinical System-New Technology MR imager (Best; Philips Medical Systems, Amsterdam, The Netherlands) with a standard quadrature head coil was used to examine map reorganization in the primary motor cortex. A $\mathrm{T}_{1}$-weighted sagittal localizer series was used to select axial slices parallel to the corpus callosum. This permitted reproducible slice selection in repeated studies. Five axial slices were selected for functional imaging to encompass the primary motor cortex. Slice thickness was $7 \mathrm{~mm}$ and the slices were contiguous. The field of view was 230 $\mathrm{mm}$, with a $128 \times 128$ imaging matrix, resulting in an inplane resolution of $1.8 \mathrm{~mm}$. For each slice, 46 dynamic time points were collected, providing a total imaging time of 3 minutes, 14 seconds for each run. The functional MR imaging sequence was a fast-field echo, multishot echo planar imaging technique with an echo planar imaging factor of 5, a repetition time of $246 \mathrm{msec}$, an echo time of $35 \mathrm{msec}$, and a flip angle of $45^{\circ}$.

The motor task consisted of biceps muscle contraction. A "block" design was used in which a cued biceps muscle contraction was alternated with a rest period for a total of three paired blocks and rest at the end of an imaging run. Patients were placed supine with both arms at rest alongside the body. The ICN-MCN reinnervated biceps muscle was contracted and relaxed repetitively, with an approximate frequency of 96 times per minute while keeping the healthy arm relaxed. The lower arm was lifted against gravity approximately $2 \mathrm{~cm}$. Patients with paralytic biceps muscles were encouraged to perform the same task, although this actually consisted of virtually rather than literally carrying out the movement. This did not result in actual biceps contraction or in limb movement. A second task consisted of contraction and relaxation of the healthy biceps muscle in a similar way, while the arm on the surgically treated side was kept relaxed. Head movement was minimized with an inflatable styrofoam-filled vacuum pillow placed around the head of the patient.

\section{Postprocessing of Images}

All images were transferred to a workstation (SUN-Sparc station; Sun Microsystems, Palo Alto, CA). Using a computer software analysis program (SPM 96, Statistical Parametric Mapping), ${ }^{9}$ all data sets were analyzed for head motion during the imaging procedure. When a data set exhibited more than a 2-mm translation in the $\mathrm{x}, \mathrm{y}$, or $\mathrm{z}$ plane during the imaging procedure, it was not used for analysis.

\section{Statistical Analysis}

The fMR imaging paradigm was a block design of three periods of alternating movement and rest during the MR imaging run (3 minutes, 14 seconds). The resulting MR imaging data set thus consisted of a matrix of $128 \times 128$ voxels with 46 sampling points. During the run the means of two groups of signal intensities (one obtained during activation and the other during rest) were calculated, with a 5-second delay to account for the delay in the hemodynamic response, which peaks at 5 to 7 seconds after the start of a task. ${ }^{2,5,29}$ Therefore, the first baseline period consisted of eight time points and subsequent periods were shifted accordingly.

Activation maps were calculated from the difference between the two means by using the Student t-test. The threshold value for statistical significance was set at 4 with a minimum cluster size of 2 , that is, at least one of eight neighboring pixels had to have a significant $t$ value as well. This threshold is based on a correction of the 0.05 significance level for spurious significance due to multiple testing, as described by Forman, et al., ${ }^{8}$ which is extrapolated to a cluster size of 2 . This criterion takes into account spatial correlations to avoid a more conservative Bonferroni correction, and has been applied successfully to detect activation in the cerebellum during intended movements. ${ }^{34}$

For comparisons across patients, active pixels were counted in a coronal strip centered over the primary motor cortex. The anterior and posterior boundaries of this strip were established at 44 and $63 \%$ of the length of the brain slice along the anteroposterior axis. During an exploratory fMR imaging experiment that involved one patient and one healthy volunteer, these boundaries were determined to be the maximum anterior and posterior extensions of the primary motor cortex. This definition was applied for all patients and all conditions.

The lateral position of the center of a cluster was measured $9 \mathrm{U}$ from the midline. The first interval on either side of the midline was not analyzed to avoid artifacts caused by draining veins, ${ }^{2}$ leaving $8 \mathrm{U}$ on either side. Thus, values 


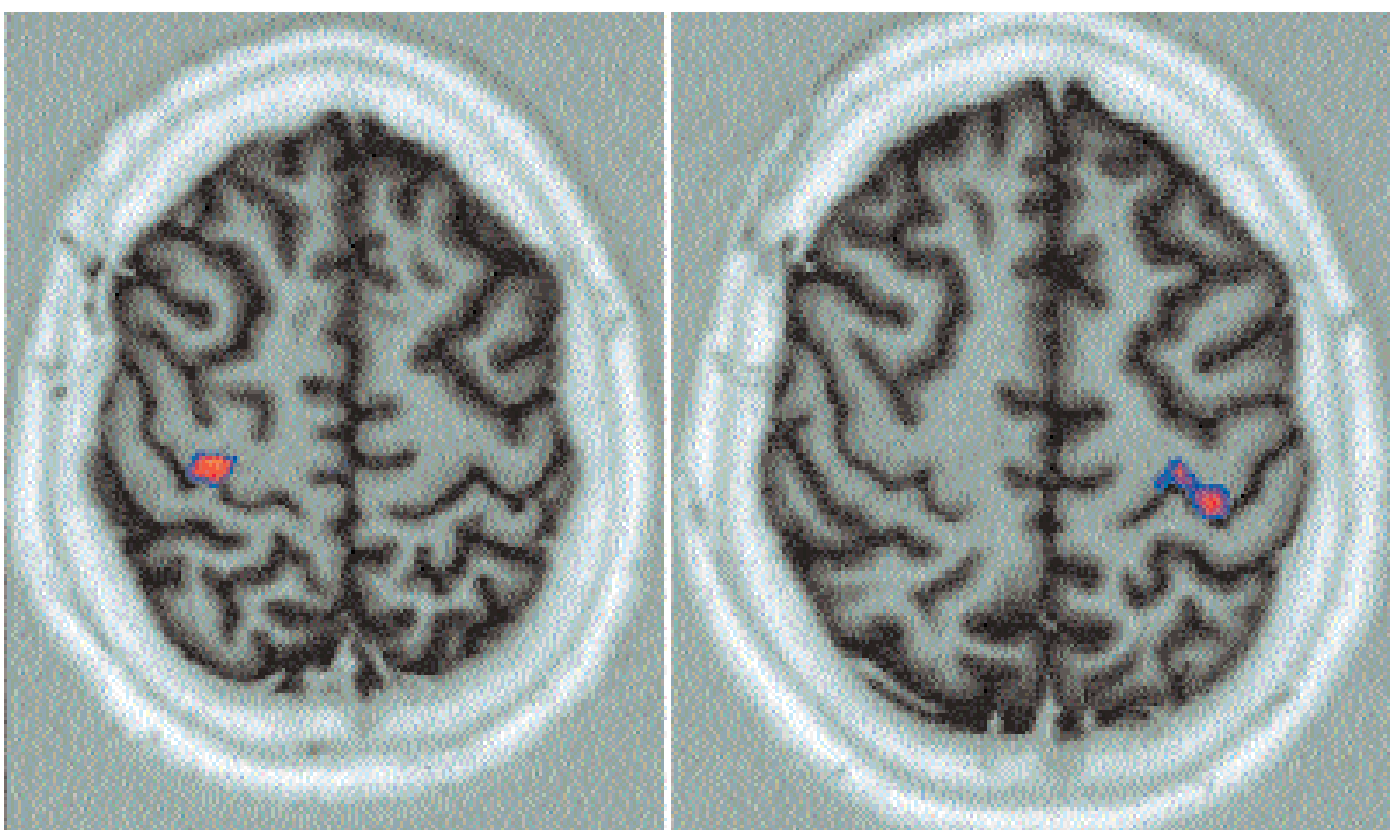

FIG. 1. Sample fMR images demonstrating activation patterns during voluntary biceps contraction. Left: Healthy side. Right: Injured side with good biceps reinnervation following ICN-MCN transfer. Functional images are overlaid on corresponding anatomical images and significant areas of activation are indicated by colored areas. Activity is localized within the primary motor area.

were obtained for a matrix of $5 \times 16 \mathrm{U}$ spanning the primary motor cortex. To compare the results for surgically treated arms that regained good biceps muscle function, arms with a paralytic biceps muscle, and control arms, the number of active pixels were counted in the corresponding maps. The ratios of active to nonactive pixels in surgically treated arms with successful biceps reinnervation and in control arms were compared using the Fisher exact text, and the mean level of activity per active pixel was compared by applying the t-test. The "center of gravity" was calculated for each condition, that is, a mean $\mathrm{x}$ and $\mathrm{y}$ coordinate was calculated for each map, in which the position of the pixels was weighted for signal density.

All mean values in this report represent means \pm SD.

\section{Results}

We used fMR imaging to test for functional reorganization in the primary motor cortex. Patients were instructed and trained in the motor task just before imaging and were monitored visually during the fMR imaging examination by the same observer throughout the entire study. Both arms were observed carefully. No mirror movements were seen in any patient during contraction of the biceps muscle. The simple biceps contractions were performed with the proximal arm well supported. Other muscle recruitment was not appreciated by the observer during training or measurement sessions. Motion artifacts were not detected in any of our data sets.

Activity could be induced and localized within the primary motor area (Fig. 1). For the seven surgically treated arms with good biceps reinnervation, active pixels were found in 28 of $80 \mathrm{U}$ in the $5 \times 16-\mathrm{U}$ matrix. For control arms, active pixels were found in 23 of $80 \mathrm{U}$ (Fig. 2). The ratio of active to nonactive units did not differ significantly between surgically treated arms with good biceps reinnervation and the control arms $(p=0.25)$. The mean level of activity for surgically treated arms was $0.47 \pm 0.51$ pixels, with a range of 0.06 to 2.63 . For control arms, the corresponding mean value was $0.55 \pm 0.58$ pixels and the range was 0.14 to 2.29 . These means did not differ significantly $(p=0.5)$. In the four patients with a flail arm, of whom two were studied before surgery and two after ICN-MCN transfer, no activity could be detected during virtual contraction of the paralytic biceps muscle. All four patients indicated independently from each other that they missed the sensation to move the arm.

For surgically treated arms that required good biceps innervation, the center of gravity lay $3.4 \mathrm{U}$ from the midline in the lateral direction and $3.25 \mathrm{U}$ in the axial direction. For control arms, these values were $3.5 \mathrm{U}$ from the midline at the contralateral side and $3.48 \mathrm{U}$ in the axial direction.

\section{Discussion}

For the patient with traumatic root avulsions of the brachial plexus, motor function can be regained using a source of axons different from those normally involved in the intended movement. The ICN-MCN transfer is a primary example of this method for restoring biceps innervation. Nevertheless, central adaptation is required to regain volitional control of elbow flexion. . $^{21,22}$

Cortical plasticity following a change in peripheral nerve circumstances has been demonstrated using TMS and fMR imaging, for example, after peripheral nerve lesion due to upper limb amputation. ${ }^{6}$ To date, however, fMR imaging has not been applied to observe the effects of nerve transfer on cerebral plasticity. In this study, we performed fMR imaging in patients with either good or absent biceps muscle 
recovery after an ICN-MCN transfer. Two patients who had a flail arm due to root avulsions of the brachial plexus were studied before surgery. Maps of motor cortex activation were generated with a biceps muscle contraction task. Activity could not be induced in patients with a paralytic biceps muscle. In those patients with good biceps muscle reinnervation, however, activity could be induced and localized within the primary motor area, where the biceps muscle previously has been mapped by direct cortical stimulation as well as by fMR imaging and positron emission tomography studies. ${ }^{15,26,27}$ Successful reinnervation seems, therefore, to be a prerequisite for the reactivation of central motor programs for volitional biceps muscle control in patients with root avulsions of the brachial plexus after ICNMCN transfer. It should be noted, however, that activation of appropriate cortical areas caused by thinking about arm movements has been found in patients totally unable to move their arms due to amyotrophic lateral sclerosis or brainstem stroke. ${ }^{1}$ Also, authors of other studies have found no significant differences in activity measured in healthy volunteers during actual and mental execution of a task..$^{30}$ The explanation of the inability to activate central motor programs in four patients in the present study remains speculative; however, it could be the case that the deafferentation in this specific patient group plays a role. Each of the four patients we studied had a flail arm and missed the feeling of being able to move the affected limb.

Neither the number of active pixels nor the mean value of their activations differed between surgically treated arms with good biceps reinnervation and control arms. The weighted center of gravity of the distribution of activity did not appear to differ either (Figs. 1 and 2), although this did not lend itself to statistical testing. There was no indication on fMR images that the neuronal input activity involved in biceps flexion after successful ICN-MCN reinnervation differed in location or intensity between surgically treated arms and control limbs. In other words, the normal cortical "biceps area" appeared to regulate biceps contraction, even though cerebral activity could not reach the biceps muscle by following the normal nervous system pathway.

\section{Location and Magnitude of the fMR Imaging Signal}

The lateral location of activity in the primary motor area that was related to biceps contraction, which was documented in this study, differs from that of the medial location of the fMR imaging signal in the motor cortex during volitional inspiration, ${ }^{7}$ which is probably related to intercostal muscle contraction. In addition, the present fMR imaging results were in exact correspondence with the results of our earlier TMS study after ICN-MCN transfer. ${ }^{21}$ In healthy volunteers it has been shown that the cortical loci corresponding to peak motor-evoked potential amplitude and peak fMR imaging activation coincide closely. ${ }^{16}$ In the current study, we found that the same applies to patients with good biceps muscle recovery after an ICN-MCN transfer.

In the report of our TMS study, we hypothesized that neural activity in the original biceps area might excite the $\alpha$-motor neurons connected to the biceps muscle through novel pathways, either in the cortex or in the spinal cord..$^{21}$ In the spinal hypothesis, new cervicothoracic connections would need to be formed between the original MCN $\alpha$-motor neurons and the original ICN $\alpha$-motor neurons. This reorganization is illustrated in Fig. 3. Theoretically, the

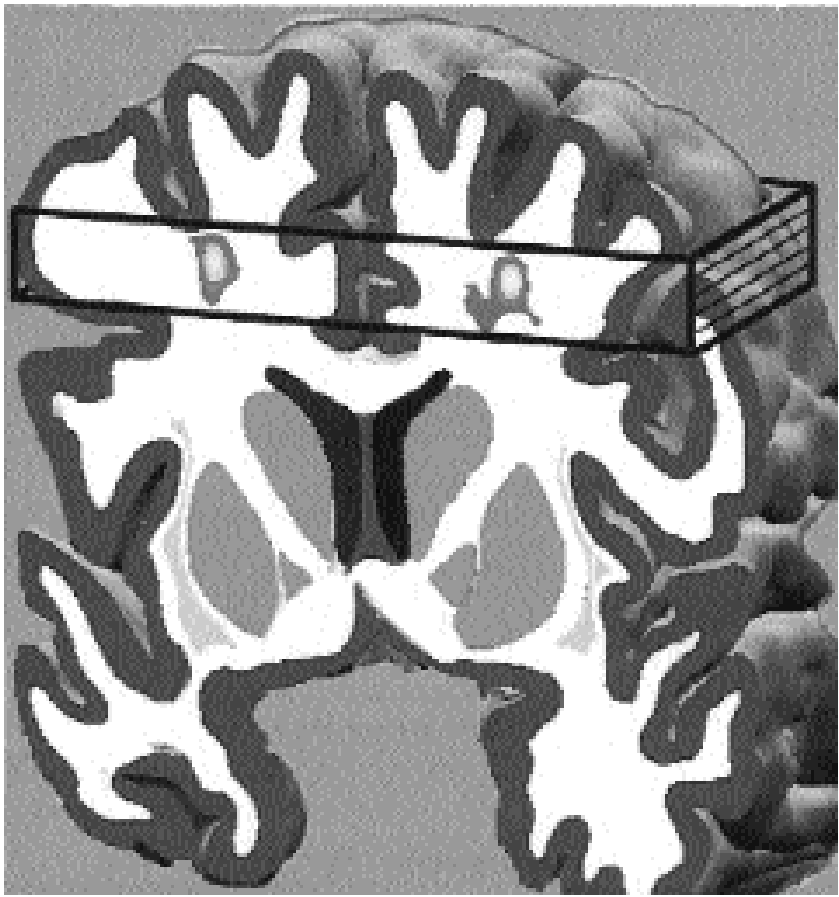

FIG. 2. Schematic representation of input activity of both control (left) and surgically treated (right) arms. The number of active pixels on axial fMR images in a coronal strip centered over the primary motor cortex were counted. The location and signal intensity of the neuronal input activity involved in biceps flexion, as measured by fMR imaging, did not differ between surgically treated arms that had regained good biceps innervation and control limbs.

corticospinal tract fibers involved after ICN-MCN transfer are either those projecting to the original $\mathrm{MCN} \alpha$-motor neurons or those projecting to the original ICN $\alpha$-motor neurons. The corticospinal biceps cells normally project to $\alpha$-motor neurons at the C-5 and C- 6 levels. Intercostal cells normally project to thoracic levels. If the corticospinal tract, which originally participated in normal biceps muscle control, remains involved after the transfer, both groups of $\alpha$-motor neurons need to be connected for functional control of the reinnervated biceps. Using retrograde nerve tracing, research in monkeys with ICN-MCN transfer has shown that this type of spinal plasticity does not occur. ${ }^{3}$ The results of two studies in humans, in which transcervical and transthoracic magnetic stimulation was used after ICNMCN transfer, confirm these findings. ${ }^{13,14}$ From both studies it can be concluded that it is unlikely that novel neural connections are formed at a spinal level. Therefore, it is expected that plastic changes in the cerebral cortex enable the restoration of function.

The magnitude of the fMR imaging signal is directly influenced by the size of the responsive area, the number of active neurons, and the degree of muscle activation. ${ }^{4,28}$ Apparently, the cortical neurons involved in biceps flexion at the end stage of successful reinnervation after ICN-MCN transfer are the same as those that are active in normal biceps flexion. From these results it could be postulated that, for the execution of biceps contraction, the denervation after trauma and subsequent ICN-MCN transfer does not seem to lead to changes in the original neural input activity and local intracortical processing. An earlier study by Gir- 


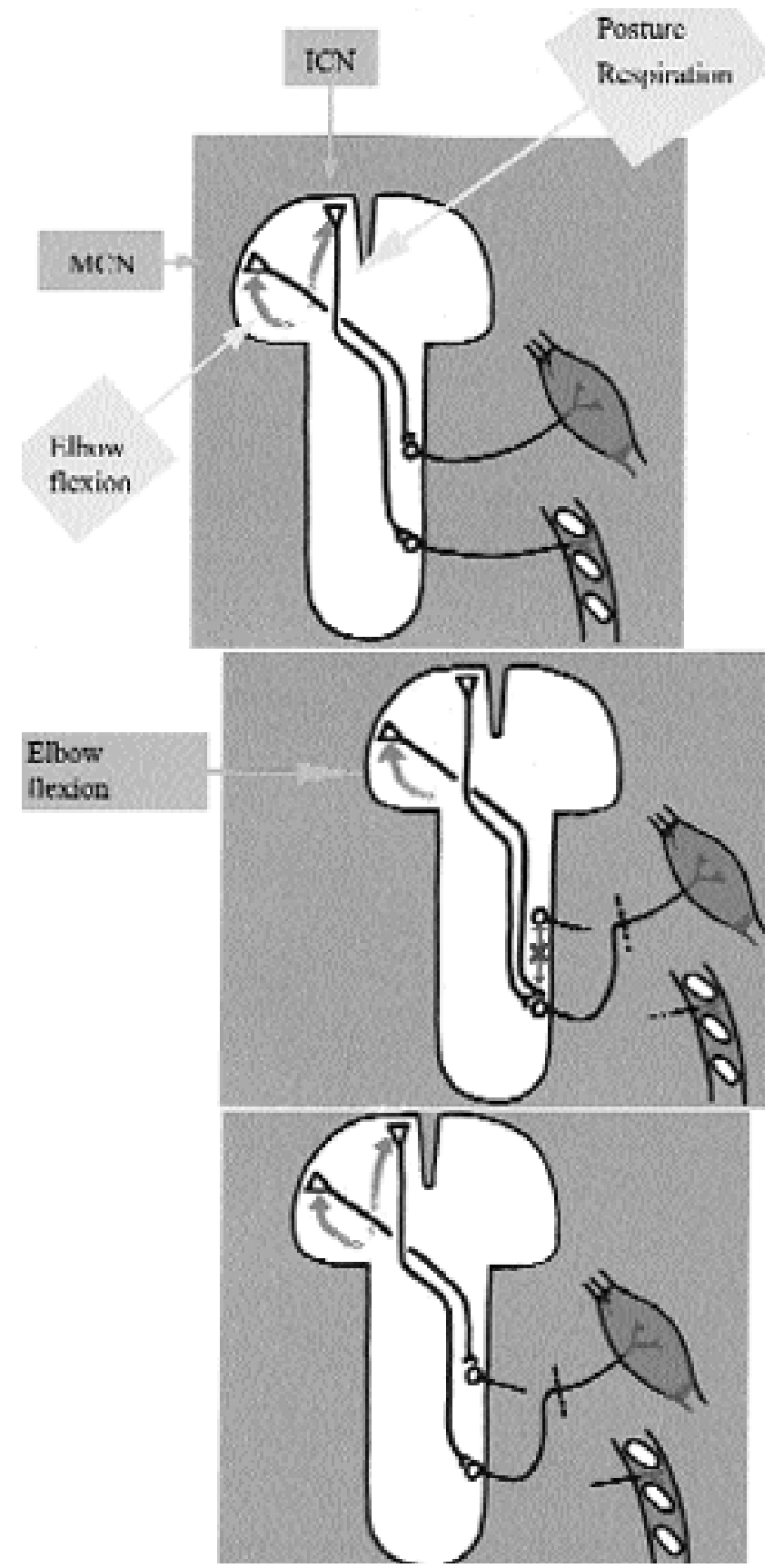

FIG. 3. Schematic representation of ICN-MCN transfer. Upper: Normal innervation. The central motor program for elbow flexion is connected to the $\mathrm{MCN}$ and the motor program for respiration and/or posture control is connected to the ICN. Center: Shortly after transfer, central ICN motor programs become connected to the biceps. Initially, biceps contraction can only be effected by means of a voluntary respiratory effort. Central adaptation is required to regain volitional control. Lower: No spinal plasticity. Neural activity in the original biceps area cannot excite ICN $\alpha$-motor neurons because novel spinal pathways have not been formed.

aux, et al., ${ }^{10}$ supports this hypothesis by the finding that, after hand transplantation, the original map for hand activation is restored, that is, the original neural network is reactivated. The new peripheral input allowed a global remodeling of the limb's cortical map and reversed the functional reorganization induced by the amputation.

With respect to the level of activation, there was a most intriguing difference between our TMS and fMR imaging data. The TMS map showed a clear difference between surgically treated patients and healthy volunteers. The cortical areas from which an ICN-MCN biceps map could be evoked were considerably smaller than areas governing the activation of control arms. Moreover, the resulting maps had lower amplitudes. ${ }^{21}$ These findings indicate that the neural output activity was decreased. This probably shows that a motor response could only be obtained from a smaller cortical neuronal population; however, a reduced number of targeted motor units may also be partially responsible for this effect. In contrast, fMR imaging demonstrated equal activity during movements of the surgically treated arm and the control arm, suggesting that similar amounts of neuronal activity occur. To explain this difference, we propose that input activity in motor function, as measured by fMR imaging, to a certain extent is independent from the output activity.

\section{Central Nervous System Plasticity After ICN-MCN Transfer}

Combining the findings in the present fMR imaging study and those from our previous TMS studies, ${ }^{20,21}$ the following concept emerges (Fig. 4). Neural input activity for volitional biceps control is reactivated following good biceps muscle reinnervation after ICN-MCN transfer. The target of the neural output activity does change, however, from the C5-6 $\alpha$-motor neuron pool to the thoracic ICN $\alpha$-motor neuron pool. One mechanism that has been suggested for mediating functional changes, is strengthening of preexisting horizontal cortical connections. ${ }^{25}$ These connections mediate the response to a common input, and enable the integrated firing of different corticospinal neuron groups of individual muscles during movement execution. It is likely that the laterally located biceps corticospinal neurons and the medially located intercostal corticospinal neurons are normally connected in this way, because coordinated activity is necessary during arm movements. ${ }^{21}$ For instance, the elbow flexion required to lift an object requires simultaneous intercostal muscle contraction for rib cage stabilization and postural control. The presence of intercortical connections between biceps and intercostal corticospinal neurons may also explain the discrepancy between the successful restoration of volitional control over biceps contraction after ICN-MCN transfer and the inability to do so after hypoglossal-MCN transfer. ${ }^{18}$ It is unlikely that connections between the tongue and upper limb cortical areas exist, in view of their isolated movement patterns. The preexisting interneuronal network for coordination of the contraction of arm and rib cage muscles involved in elbow flexion thus creates an accessible output pathway for the control of the reinnervated biceps muscle.

\section{Conclusions}

From this fMR imaging study it can be concluded that successful biceps muscle reinnervation after ICN-MCN transfer induces the reactivation of central motor programs. The cortical input for voluntary biceps contraction at the 


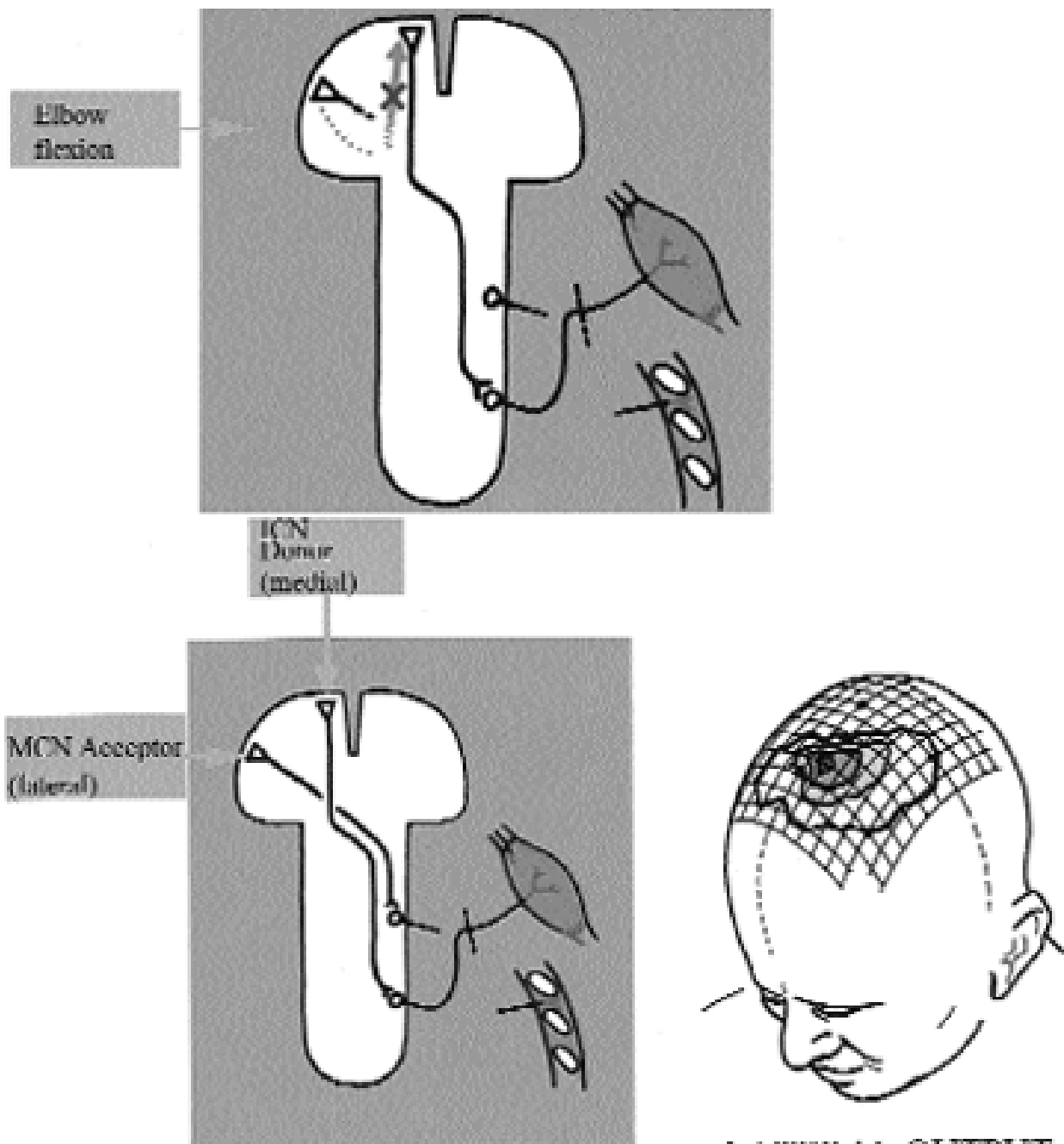

Luleral Uutput. less cocilitite, smaller (TMS)

\section{LATERAL OUTTITT}

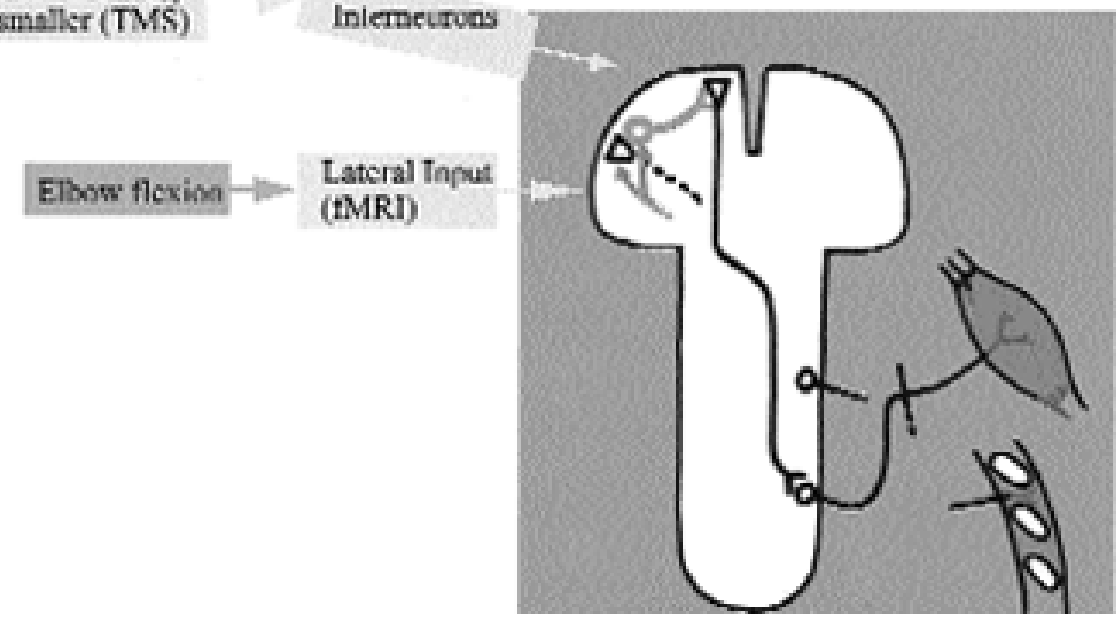

FIG. 4. Schematic representation of the concept of central nervous system plasticity after ICN-MCN transfer, based on the present fMR imaging study and previous TMS studies. Upper: Neural input activity for volitional biceps control does not show plastic changes after ICN-MCN transfer according to findings. Center: The center of gravity of the ICN-MCN biceps TMS map is indistinguishable from that of the normal biceps muscle location and is different from that of the medial location of the intercostal muscles. These findings show that plastic changes occur in the neural output activity. Lower: An interneuronal network between the ICN donor and the MCN acceptor creates an accessible output pathway by mediating the response to a common input. 
end stage of reinnervation does not show plastic changes and has MCN acceptor qualities. Apparently, plastic changes after ICN-MCN transfer are limited to the output neural activity. The presence of a common input response between the corticospinal neurons of the ICN donor and the MCN acceptor seems crucial to obtain a functional result after transfer. Moreover, it may even be the case that a common input response between donor and acceptor needs to be present in all types of nerve transfer to become functionally effective.

\section{References}

1. Bakay RAE: Comment on Roux FE, Ibarrola D, Lazorthes Y, et al: Virtual movements activate primary sensorimotor areas in amputees: report of three cases. Neurosurgery 49:736-742, 2001

2. Bandettini PA, Jesmanowicz A, Wong EC, et al: Processing strategies for time-course data sets in functional MRI of the human brain. Magn Reson Med 30:161-173, 1993

3. Cheng H, Shoung HM, Wu ZA, et al: Functional connectivity of the transected brachial plexus after intercostal neurotization in monkeys. J Comp Neurol 380:155-163, 1997

4. Dai TH, Liu JZ, Sahgal V, et al: Relationship between muscle output and functional MRI-measured brain activation. Exp Brain Res 140:290-300, 2001

5. Dale AM, Buckner RL: Selective averaging of rapidly presented individual trials using fMRI. Hum Brain Mapp 5:329-340, 1997

6. Dettmers C, Liepert J, Adler T, et al: Abnormal motor cortex organization contralateral to early upper limb amputation in humans. Neurosci Lett 263:41-44, 1999

7. Evans KC, Shea SA, Saykin AJ: Functional MRI localisation of central nervous system regions associated with volitional inspiration in humans. J Physiol 520:383-392, 1999

8. Forman SD, Cohen JD, Fitzgerald M, et al: Improved assessment of significant activation in functional magnetic resonance imaging (fMRI): use of a cluster-size threshold. Magn Reson Med 33: 636-647, 1995

9. Friston KJ, Frith CD, Liddle PF, et al: Comparing functional (PET) images: the assessment of significant change. J Cereb Blood Flow Metab 11:690-699, 1991

10. Giraux P, Sirigu A, Schneider F, et al: Cortical reorganization in motor cortex after graft of both hands. Nat Neurosci 4:691-692, 2001

11. Hallett M: Transcranial magnetic stimulation and the human brain. Nature 406:147-150, 2000

12. Jasper HH: Report of the Committee on Methods of Clinical Examination in Electroencephalography. Electroencephalogr Clin Neurophysiol 10:370-375, 1958

13. Kawai $\mathrm{H}$ : Intercostal nerve transfer, in Kawai $\mathrm{H}$, Kawabata $\mathrm{H}$ (eds): Brachial Plexus Palsy. Singapore: World Scientific, 2000, pp 161-236

14. Kawai H, Murase T, Shibuya R, et al: Magnetic stimulation of biceps after intercostal cross-innervation for brachial plexus palsy. A study of motor evoked potentials in 25 patients. J Bone Joint Surg (Br) 76:666-669, 1994

15. Kawashima R, Itoh H, Ono S, et al: Activity in the human primary motor cortex related to arm and finger movements. Neuroreport 6:238-240, 1995

16. Krings T, Buchbinder BR, Butler WE, et al: Functional magnetic resonance imaging and transcranial magnetic stimulation: com- plementary approaches in the evaluation of cortical motor function. Neurology 48:1406-1416, 1997

17. Logothetis NK, Pauls J, Augath M, et al: Neurophysiological investigation of the basis of the fMRI signal. Nature 412:150-157, 2001

18. Malessy MJA, Hoffmann CF, Thomeer RTWM: Initial report on the limited value of hypoglossal nerve transfer to treat brachial plexus root avulsions. J Neurosurg 91:601-604, 1999

19. Malessy MJA, Thomeer RTWM: Evaluation of intercostal to musculocutaneous nerve transfer in reconstructive brachial plexus surgery. J Neurosurg 88:266-271, 1998

20. Malessy MJA, Thomeer RTWM, van Dijk JG: Changing central nervous system control following intercostal nerve transfer. J Neurosurg 89:568-574, 1998

21. Malessy MJA, van der Kamp W, Thomeer RTWM, et al: Cortical excitability of the biceps muscle after intercostal-to-musculocutaneous nerve transfer. Neurosurgery 42:787-795, 1998

22. Malessy MJA, van Dijk JG, Thomeer RTWM: Respiration-related activity in the biceps brachii muscle after intercostal-musculocutaneous nerve transfer. Clin Neurol Neurosurg 95 (Suppl): S95-S102, 1993

23. Malessy MJA, van Duinen SG, Feirabend HKP, et al: Correlation between histopathological findings in C-5 and C-6 nerve stumps and motor recovery following nerve grafting in brachial plexus repair. J Neurosurg 91:636-644, 1999

24. Mano Y, Nakamuro T, Tamura R, et al: Central motor reorganization after anastomosis of the musculocutaneous and intercostal nerves following cervical root avulsion. Ann Neurol 38:15-20, 1995

25. Nudo RJ, Plautz EJ, Frost SB: Role of adaptive plasticity in recovery of function after damage to motor cortex. Muscle Nerve 24: 1000-1019, 2001

26. Penfield W, Jasper H: Epilepsy and the Functional Anatomy of the Human Brain. Boston: Little, Brown, 1954, pp 52-77

27. Rao SM, Binder JR, Hammeke TA, et al: Somatotopic mapping of the human primary motor cortex with functional magnetic resonance imaging. Neurology 45:919-924, 1995

28. Rees G, Friston K, Koch C: A direct quantitative relationship between the functional properties of human and macaque V5. Nat Neurosci 3:716-723, 2000

29. Rosen BR, Buckner RL, Dale AM: Event-related functional MRI: past, present, and future. Proc Natl Acad Sci USA 95:773-780, 1998

30. Roth M, Decety J, Raybaudi M, et al: Possible involvement of primary motor cortex in mentally simulated movement: a functional magnetic resonance imaging study. Neuroreport 7:1280-1284, 1996

31. Seddon HJ (ed): Peripheral Nerve Injuries. London: HM Stationery Office, 1954

32. Thomeer RTWM, Malessy MJA: Surgical repair of brachial plexus injury. Clin Neurol Neurosurg 95 (Suppl):S65-S72, 1993

33. Wassermann EM, McShane LM, Hallett M, et al: Noninvasive mapping of muscle representations in human motor cortex. Electroencephalogr Clin Neurophysiol 85:1-8, 1992

34. Xue M, Ng TC, Yue G, et al: Activation of cerebellar cortex during intended motor actions. Proc Soc Magn Res:447, 1996 (Abstract)

Manuscript received April 26, 2002.

Accepted in final form September 9, 2002.

Address reprint requests to: Martijn J. A. Malessy, M.D., Ph.D., Department of Neurosurgery, Leiden University Medical Center, P. O. Box 9600, 2300 RC Leiden, The Netherlands. email: M.J.A. Malessy@lumc.nl. 